\title{
MODELAGEM CINÉTICA DA REAÇÃO DE HIDRÓLISE ÁCIDA DO AMIDO EM MEIO ACÉTICO
}

\author{
D. V. M. LOPES ${ }^{1}$, B. F. SANTOS ${ }^{1}$, E. B. M. MEDEIROS ${ }^{1}$ e N. M. LIMA FILHO ${ }^{1}$ \\ ${ }^{1}$ Universidade Federal de Pernambuco, Departamento de Engenharia Química \\ E-mail para contato: deyvidvml@gmail.com
}

RESUMO - O presente trabalho teve como objetivo, realizar o estudo cinético da hidrólise ácida do amido na presença de um ácido orgânico, fazendo uso da hipótese de um modelo cinético fenomenológico e dados experimentais. As reações foram efetuadas em reator do tipo batelada com agitação mecânica nas seguintes condições operatórias: temperaturas compreendidas entre 136 a $176^{\circ} \mathrm{C}, \mathrm{P}=10 \mathrm{~atm}$, sistema fechado $\left(\mathrm{N}_{2}\right)$, massa do amido $75 \mathrm{~g}$, volume da solução de ácido acético $750 \mathrm{ml}$, tempo máximo de reação 4 horas, concentração do ácido 2,5g/l e agitação de 500rpm. Os resultados experimentais foram satisfatórios, na temperatura de $176^{\circ} \mathrm{C}$ obtemos a produção máxima em glicose em torno de 42,06g/L. Resultante dos dados experimentais a possibilidade de postular a hipótese de um modelo cinético fenomenológico e valida-lo, considerando o estado estacionário complexo para os produtos intermediários instáveis obtendo-se uma função objetiva de ordem $10^{-2}$, proporcionando a quantificação da constante de velocidade da reação e a constante de dissociação do ácido acético.

\section{INTRODUÇÃO}

Os sacarídeos constituem uma classe de compostos que podem ser funcionalizados ou degradados resultando em substâncias passíveis de diversas aplicações. Estes aspectos somados as suas disponibilidades, a partir de fontes vegetais agrícolas, os qualificam como produtos possuidores de grande potencial de valorização (Lima Filho, 1996; Keikhosro et al., 2006; HuaJiang et al., 2008), através de reações de oxidação, hidrogenação, acetilação, esterificação, etc., na obtenção de compostos de comprovadas utilidades industriais.

Abordando as peculiaridades relativas à reatividade do polissacarídeo (amido) em meio ácido, empreendeu-se no presente trabalho o desenvolvimento do processo de hidrólise em presença de um catalisador homogêneo (ácido acético), procurando direcionar para futuros estudos a possibilidade de produção de sorbitol em uma só etapa hidrólise-hidrogenação em presença de um segundo catalisador suportado (Oliveira, 2004).

Como se sabe os principais constituintes do amido são $\alpha$-amilose polímero constituído de mais de 6000 unidades de $\alpha$-D-glicose (cadeia linear de ligações 1-4 de glicosídica), Figura 1.

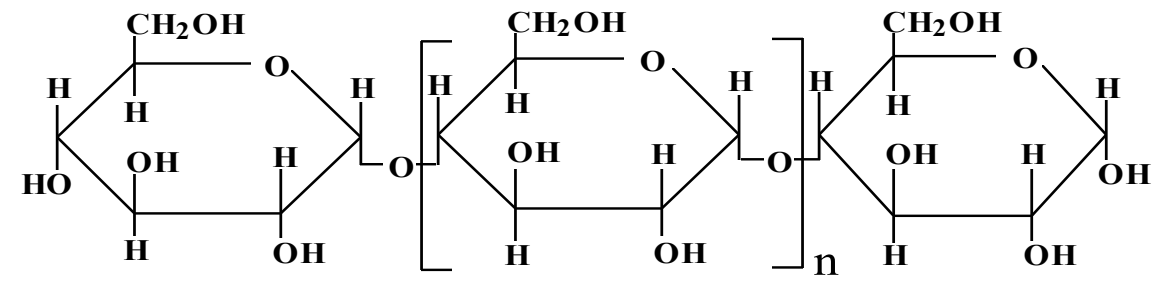

Figura 1 - Estrutura molecular da cadeia de $\alpha$-amilose. 
E a amilopectina, molécula grande e altamente ramificada, com massa molecular médio da ordem de 107-109. É formada por várias cadeias constituídas de 20-25 unidades de $\alpha$-D-glicose com ligações em $\alpha$-(1-4) e igualmente $\alpha$ - (1-6) constituindo essas ligações de 4-5\% do total das ligações glicosídicas, conforme mostrada na Figura 2 (PERONI, 2003).

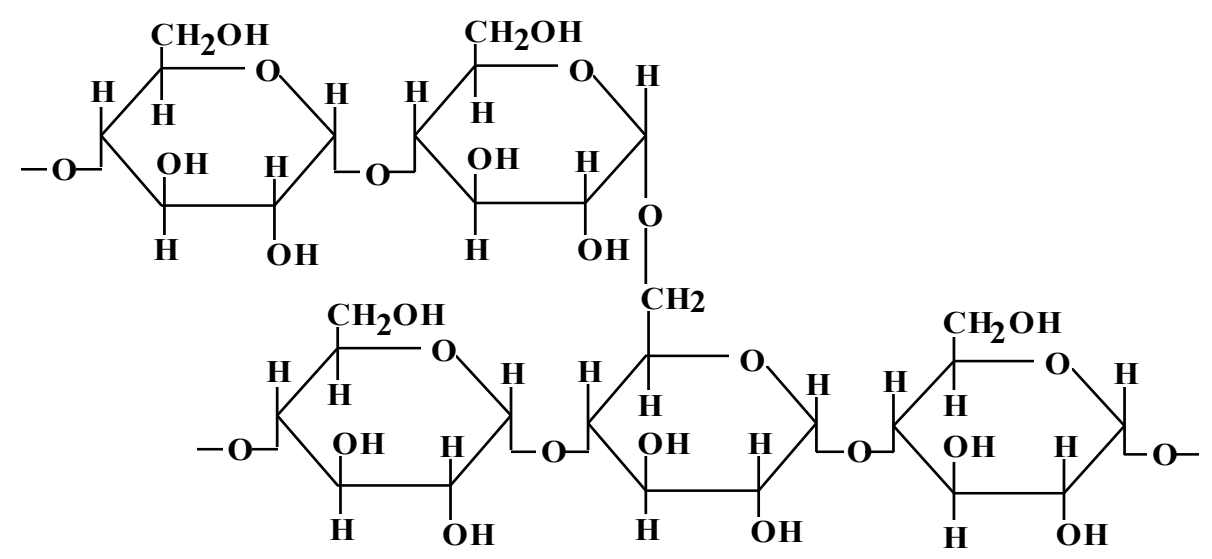

Figura 2 - Estrutura molecular da cadeia de amilopectina.

A reação de hidrólise ácida direta do amido seguida da reação de hidrogenação catalítica, com a obtenção de polióis como sorbitol, maltotriol e maltitol, ainda não é aplicada nos processos industriais convencionais e precisa ser estudada, pois é de grande importância para as futuras biorrefinarias dedicadas. Neste contexto o presente trabalho apresenta $o$ desenvolvimento de modelos cinéticos ligados à possibilidade do processo de hidrólise ácida associada em duas etapas ou em uma única etapa, para produção catalítica de hidrogenados.

\section{MATERIAIS E MÉTODOS}

\subsection{Materiais}

Como parte do estudo sistemático do processo de hidrólise ácida do amido, as experiências recorreram os seguintes materiais: reagentes (ácido acético VETEC PA, fécula de mandioca da empresa NEUPEL S.A), reator de aço inox Modelo PARR, Cromatografo Líquido de Altas Eficiências - CLAE com detecção por índice de refração e coluna HPX-87H.

\subsection{Procedimento Experimental}

Os experimentos foram realizados em um reator trifásico do tipo agitado mecanicamente (modelo PARR 3543), Figura 3, processando-se suspensões aquosas de amido, com concentração inicial de $75 \mathrm{~g} / \mathrm{L}$ em presença de meio acético. O reator de aço inoxidável foi aquecido eletricamente segundo um programador controlador de temperatura PID até a temperatura de processamento, operando em batelada para as duas fases envolvidas no processo: líquida (suspensão de amido) e gasosa $\left(\mathrm{N}_{2}\right)$. O nitrogênio é introduzido através de uma válvula no topo do reator mantendo-se a pressão do mesmo constante (10bar) com ajuda de um transdutor e uma válvula de passagem de gás. A temperatura de reação é medida através 
de termopares e regulada segundo controladores específicos. Após o estabelecimento da temperatura e pressão da reação iniciou-se a coleta da amostra, tomando-se como tempo de processamento inicial " $\mathrm{t}_{0}$ " o tempo a partir do momento que o mesmo atingir a temperatura e pressão de operação. Em seguida dá-se início a contagem do tempo de operação, mantendo controlada a temperatura e pressão total.

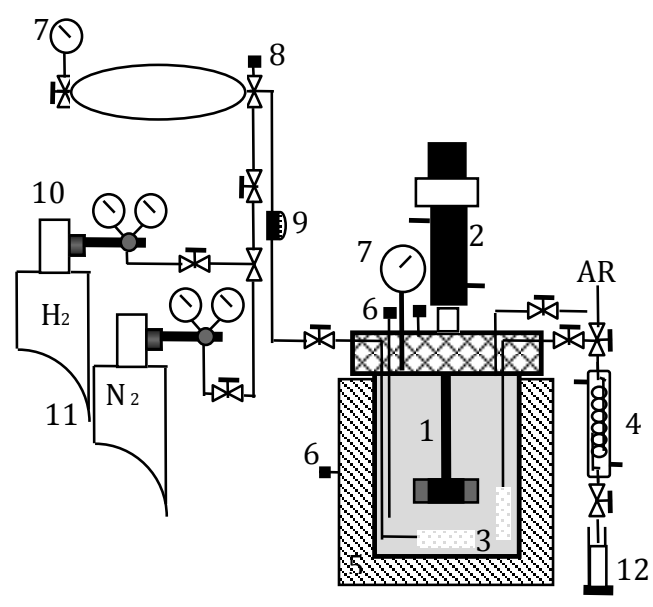

1. Reator, 2. Agitador, 3. Distribuidor de gás, 4. Sistema coletor da amostra, 5. Forno de aquecimento, 6. Termopares, 7. Manômetros, 8. Válvulas, 9. Válvulas reguladoras da pressão, 10. Manômetros reguladores de pressão, 11. Cilindros, 12. Coletor de amostra.

Figura 3 - Reator utilizado na Hidrólise Ácida do Amido de Mandioca.

A cada 30 minutos foram coletados $5 \mathrm{ml}$ de amostra a partir de um difusor poroso imerso no seio do líquido, em seguida as amostras coletadas são preparadas com a diluição adequada, filtradas e levadas diretamente às análises por cromatografia liquida de altas eficiências com detecção por índice de refração, utilizando uma coluna AMINEX HPX 87P, tendo-se como fase móvel água permutada e filtrada com a vazão de $0,6 \mathrm{~mL} / \mathrm{min}$. A coluna foi mantida a $80^{\circ} \mathrm{C} \pm 1^{\circ} \mathrm{C}$ e foram identificados e quantificados os produtos provenientes das reações de hidrólise.

\section{RESULTADOS E DISCUSSÃO}

Ensaios preliminares foram realizados inicialmente a níveis de testes, com ácido acético em temperaturas compreendidas entre 90 a $100^{\circ} \mathrm{C}$ e pressão atmosférica e em refluxo, sistema que não permitiu a avaliação com o ácido acético, devido a necessidade do aumento da temperatura de operação.

No intuito de se validar a possibilidade da utilização do ácido acético no processo de hidrólise ácida do amido, optou-se por um sistema mais adequado a utilização de temperaturas aplicadas compreendidas entre 136 a $176^{\circ} \mathrm{C}$, considerando o aumento da pressão em sistema fechado, e com auxílio de um gás inerte nitrogênio, para manter a solução em fase líquida e não vapor.

Os resultados experimentais obtidos nas três temperaturas utilizadas na hidrólise ácida do amido, tomado como reagente modelo, Figura 4, apresenta comportamento progressivo com o aumento da temperatura, confirmando os efeitos efetivos causados pela mesma sobre a dissociação do ácido acético. 


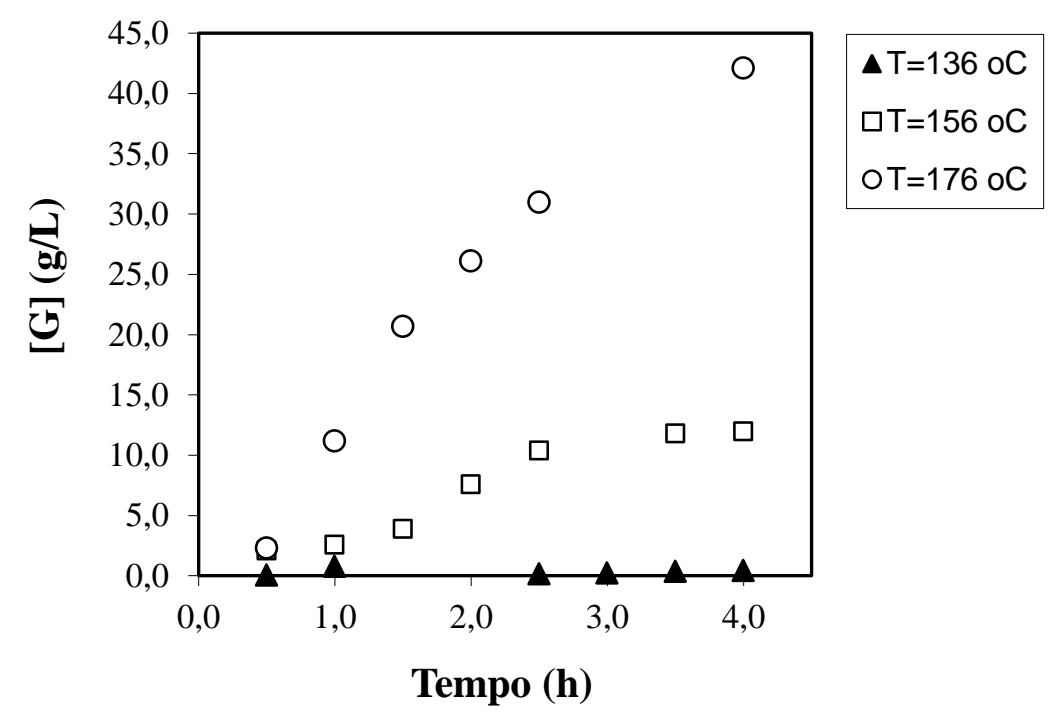

Figura 4 - Evolução cinética da produção de glicose em meio acético

$\mathrm{O}$ aumento da temperatura em torno de $176^{\circ} \mathrm{C}$ foi relevante na evolução da reação de hidrólise do amido, comprovando a papel favorável do aumento da presença de íons $\mathrm{H}^{+}$no meio reacional para realização da reação de hidrólise.

\subsection{Influência da presença do Ácido Acético e da Temperatura}

A necessidade de otimizar o sistema operacional do processo de hidrólise está associada aos efeitos da dissociação do ácido acético em meio aquoso, por este ser um ácido fraco, também aos efeitos da temperatura do processo de hidrólise ácida. Assim o conhecimento de um modelo fenomenológico que possa representar corretamente a degradação do amido em glicose e seus oligossacarídeos, deve partir a princípio da dissociado do ácido acético, representado nas equações 1 e 2 .

$$
\begin{aligned}
& H A C \rightleftarrows A C^{-}+H^{+} \\
& H^{+}+H_{2} O \rightleftarrows H_{3} O^{+}
\end{aligned}
$$

Partindo do conhecimento da constante de dissociação do ácido acético em meio aquoso de acordo com a equação 3 .

$$
K_{A C^{-}}=\frac{K_{A}}{K_{-A}}=\frac{\left[A C^{-}\right]\left[H^{+}\right]}{[H A C]} ;\left[H^{+}\right] \cong\left[H_{3} O^{+}\right] \cong\left[A C^{-}\right]
$$

Desta maneira, procurou-se encontrar a temperatura adequada de operação para o processo, objetivando a minimização do tempo de reação e o crescimento do rendimento em produtos hidrolisados. Logo envolve a constante de dissociação, como a melhor maneira de representar a influência deste parâmetro operacional sob a reação de hidrólise ácida. 


$$
K_{A C^{-}}=\frac{\left[H^{+}\right]^{2}}{[H A C]} \quad ;\left[H^{+}\right]=\left([H A C] K_{A C^{-}}\right)^{\frac{1}{2}} ; K_{A C^{-}}=f(T)
$$

Partindo do princípio da equação de Henderson-Hassel, tem-se o valor da concentração de íons acetato $\left[A C^{-}\right]$.

$$
p H=p K a+\log \frac{\left[A C^{-}\right]}{[H A C]}
$$

$\mathrm{O}$ pH inicial medido para solução de ácido acético foi 3,32 para concentração de ácido acético [HAC] de 0,4717 M, o pKa tabelado é de 4,76. Logo teremos:

$$
3,32-4,76=\log \frac{\left[A C^{-}\right]}{0,417}
$$

Daí o valor calculado da concentração de $\left[A C^{-}\right]$, a partir da equação (6) é 1,66× $10^{-2}$.

E a partir do valor calculado da concentração de $\left[A C^{-}\right]$, determinou-se $K_{A C^{-}}$, sabendo-se que $\left[H^{+}\right] \cong\left[A C^{-}\right]$temos:

$$
K_{A C^{-}}=\frac{\left[H^{+}\right]^{2}}{[H A C]}=\frac{\left(1,6610^{-2}\right)^{2}}{0,417}=6,61 \times 10^{-4}
$$

\subsection{Mecanismo Cinético da Hidrólise Ácida do Amido}

Aplicado à hipótese da dissociação total do ácido acético, segundo os efeitos da temperatura. Postulou-se uma hipótese de mecanismo cinético fenomenológico, que considera as etapas de consumo e formação dos oligossacarídeos para a produção de glicose, conforme mecanismo seguinte.

$$
\begin{gathered}
\mathrm{AMD}+\mathrm{H}_{3} \mathrm{O}^{+} \rightleftarrows A M D^{*} \\
A M D^{*} \rightarrow D P n+G \\
D P n+H_{3} O^{+} \rightleftarrows D P n^{*} \\
D P_{n}^{*} \rightarrow D P_{n-1}+G \\
D P_{n-1}+H_{3} O^{+} \rightleftarrows D P_{n-1}^{*} \\
D P_{n-1}^{*} \rightarrow D P_{n-2}+G
\end{gathered}
$$

Considerando todas as etapas reacionais de primeira ordem, e obedecendo a lei de velocidade segundo a equação (8). 


$$
r_{G}=k_{2}\left[A M D^{*}\right]+k_{3}\left[D P_{n}^{*}\right]+k_{4}\left[D P_{n-1}^{*}\right]+\cdots
$$

E aplicando a teoria de aproximação do estado estacionário aos intermediários instáveis $\left[A M D^{*}\right],\left[D P_{n}^{*}\right] \mathrm{e}\left[D P_{n-1}^{*}\right]$, além da consideração de hipótese de cinética de primeira ordem, têm-se.

$$
\begin{gathered}
\frac{d\left[A M D^{*}\right]}{d t}=k_{1}[\mathrm{AMD}]\left[H_{3}^{+} O\right]-k_{-1}\left[A M D^{*}\right]-k_{2}\left[A M D^{*}\right] \approx 0 \\
\frac{d\left[D P_{n}^{*}\right]}{d t}=k_{1}^{\prime}\left[D P_{n}\right]\left[H_{3}^{+} O\right]-k_{-1}^{\prime}\left[D P_{n}^{*}\right]-k_{3}\left[D P_{n}^{*}\right] \approx 0 \\
\frac{d\left[D P_{n-1}^{*}\right]}{d t}=k_{2}^{\prime}\left[D P_{n-1}\right]\left[H_{3}^{+} O\right]-k_{-2}^{\prime}\left[D P_{n-1}^{*}\right]-k_{4}\left[D P_{n-1}^{*}\right] \approx 0
\end{gathered}
$$

Reorganizando as equações (9), (10) e (11) e substituindo $\left[\mathrm{H}_{3}^{+} \mathrm{O}\right]$ nas respectivas equações, resulta em:

$$
\begin{gathered}
{\left[A M D^{*}\right]=\frac{k_{1}[A M D]\left([H A C] K_{A C^{-}}\right)^{1 / 2}}{\left(k_{-1}+k_{2}\right)}} \\
{\left[D P_{n}^{*}\right]=\frac{k_{1}^{\prime}\left[D P_{n}\right]\left([H A C] K_{A C^{-}}\right)^{1 / 2}}{\left(k_{-1}^{\prime}+k_{3}\right)}} \\
{\left[D P_{n-1}^{*}\right]=\frac{k_{2}^{\prime}\left[D P_{n-1}\right]\left([H A C] K_{A C^{-}}\right)^{1 / 2}}{\left(k_{-2}^{\prime}+k_{4}\right)}}
\end{gathered}
$$

Considerando o monitoramento final da reação pela taxa de produção de glicose tem-se:

$r_{G}=\left([H A C] K_{A C^{-}}\right)^{1 / 2}\left\{\frac{k_{2}[A M D]}{\left(k_{-1}+k_{2}\right) / k_{1}}+\frac{k_{3}\left[D P_{n}\right]}{\left(k_{-1}^{\prime}+k_{3}\right) / k_{1}^{\prime}}+\frac{k_{4}\left[D P_{n-1}\right]}{\left(k_{-2}^{\prime}+k_{4}\right) / k_{2}^{\prime}}+\ldots\right\}$

Como $k_{1}=k_{1}^{\prime}=k_{2} ; k_{-1}=k_{-1}^{\prime}=k_{2} ; k_{2}=k_{3}=k_{4}$, adota - se:

$$
k=\frac{k_{2}}{\left(k_{-1}+k_{2}\right) / k_{1}}=\frac{k_{3}}{\left(k_{-1}^{\prime}+k_{3}\right) / k_{1}^{\prime}}=\frac{k_{4}}{\left(k_{-2}^{\prime}+k_{4}\right) / k_{2}^{\prime}}=\cdots=\cdots
$$

Substituindo a relação obtida para k na equação (15), tem-se, 


$$
r_{G}=k\left([H A C] K_{A C^{-}}\right)^{\frac{1}{2}}\left\{[A M D]_{0}-[G]\right\}
$$

Considerando como condição inicial que em $\mathrm{t}=0 ;[A M D]=[A M D]_{0}$ e monitorando a concentração da formação de glicose ao longo do tempo de reação, aplicou-se um tratamento computacional para validação do modelo cinético fenomenológico proposto.

Partindo do balanço material dos reagentes e produtos do meio reacional, observa-se a equação diferencial como representativa da evolução cinética do processo de hidrólise em meio acético contendo a suspensão de amido, a partir do inicio da operação isotérmica, $\mathrm{t}_{0}, \mathrm{Sendo}_{0} \mathrm{o}$ tempo a qual a mistura reacional atinge a temperatura e pressão da reação inicial de reação.

A equação diferencial obtida do modelo proposto foi aplicado o procedimento de otimização recorrendo à minimização de uma função objetivo $\left(f_{0}\right)$ definida como a diferença quadrática entre seus valores de concentrações experimentais e calculados do modelo, $f_{0}=\sum\left([\mathrm{G}]_{\mathrm{i} \text { exp }}-[\mathrm{G}]_{\mathrm{i}, \text { calc }}\right)^{2}$, com o auxílio do método de integração numericamente do tipo Runge-Kutta de $4^{\mathrm{a}}$ ordem, de forma a obter-se valores das duas constantes envolvidas no processo.

A ferramenta computacional utilizada para os cálculos foi o software Visual Fortran versão 6.2. Os valores das concentrações experimentais e calculadas são apresentados na Figura 5, já os respectivos valores das constantes envolvidas no processo de hidrólise ácida são mostrados na Tabela 1 , para a temperatura de $176^{\circ} \mathrm{C}$.

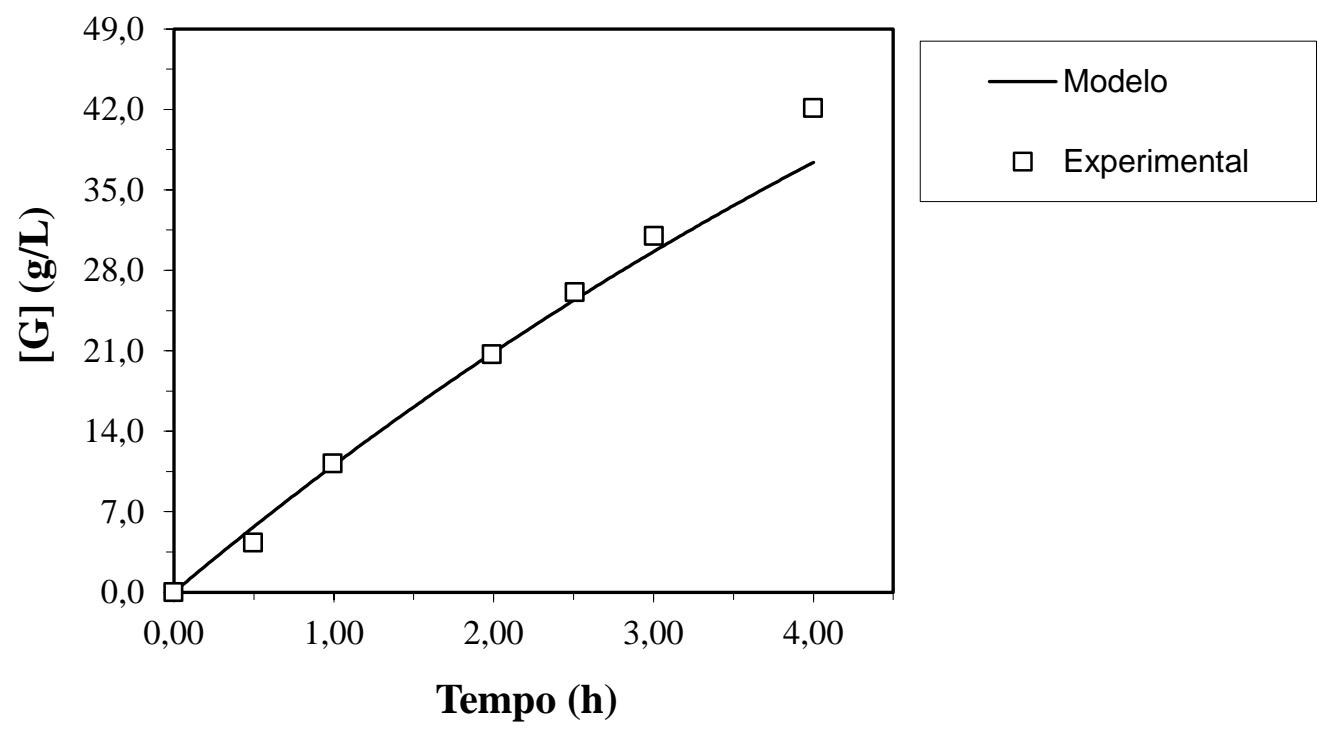

Figura 5- Modelagem Cinética da Hidrólise Ácida do Amido em Meio Acético. Condições operacionais: $\mathrm{T}=176^{\circ} \mathrm{C} ; \mathrm{V}_{\mathrm{L}}=0,75 \times 10^{-3} \mathrm{dm}^{3} ;[A M D]_{0}=75 \mathrm{~g} / \mathrm{L}$. 
Tabela 1 - Constante de Velocidade da Reação de Hidrólise Ácida na Presença do Ácido Acético. Erro médio de 9,40\%.

\begin{tabular}{|c|c|}
\hline $\begin{array}{c}\text { Constante de } \\
\text { Velocidade }\end{array}$ & Temperatura $\left({ }^{\circ} \mathrm{C}\right)$ \\
\cline { 2 - 2 } & $\mathrm{T}_{176}$ \\
\hline \hline $\mathrm{k}\left(\mathrm{min}^{-1}\right)$ & 0,0301 \\
\hline$K_{A C^{-}}$ & 0,00168 \\
\hline
\end{tabular}

\section{CONCLUSÃO}

A reação de hidrolise ácida da fécula do amido de mandioca na temperatura de $176^{\circ} \mathrm{C}$ apresentou rendimento em glicose de $42,06 \%$, nas condições operacionais: $V_{L}=0,75 \times 10^{-3} \mathrm{dm}^{3}$ e $[A M D]_{0}=75 \mathrm{~g} / \mathrm{L}$, pressão total de $10 \mathrm{bar}$.

A influência da temperatura é verificada tal que um mínimo de $136^{\circ} \mathrm{C}$, não permite um avanço efetivo da reação no sentido da forma da glicose, com rendimento inferior aos $5 \%$ da mesma. Além da necessidade da utilização do aumento da pressão para realização da coleta de amostra. $\mathrm{O}$ aumento da temperatura para $176^{\circ} \mathrm{C}$ promoveu um aumento da ordem de 2,5 na constante de dissociação do ácido acético, determinada a partir da modelagem matemática.

O modelo cinético proposto, considerando estado estacionário complexo para os produtos intermediários instáveis ajustou-se aos resultados experimentais, obtendo-se uma função objetiva de ordem $10^{-2}$, proporcionando a quantificação da constante de velocidade da etapa de hidrólise ácida e a constante de dissociação responsável pela etapa de aumento de íons $\mathrm{H}^{+}$no meio reacional, comprovada com o aumento da temperatura do meio reacional.

\section{REFERÊNCIAS BIBLIOGRÁFICAS}

LIMA FILHO, N. M., "Hydrogénolyse catalytique d'amidon de maïs en réacteur gaz-liquidesolide mécaniquement agité", Thèse de Doctorat INPL (1996), Nancy, France.

KEIKHOSRO, K., SHAUKER, K., MOHAMMAD, J. T., Conversion of rice straw to sugars by dilute-acid hydrolysis, Biomass and Bioenergy, 30 (2006) 247-253.

HUA-JIANG, H.; RAMASWAMY, S.; TSCHIRNER, S.; RAMARAO, B. V., A review of separation Technologies in current and future biorefineries, Separation and Purification Technology, 62, 1-21, 2008.

OLIVEIRA, T. A., Valorização de Resíduos Amiláceos por Hidrogenação Catalítica. Modelagem Cinética do Processo. Recife, Brasil, UFPE, 2004, (Dissertação de Mestrado).

PERONI, F. H. G. Características estruturais e físico-químicas de amidos obtidos de diferentes fontes botânicas, 118f. Dissertação (Mestrado em Engenharia e Ciência de Alimentos) - Instituto de Biociências, Letras e Ciências Exatas, São José do Rio Preto, 2003. 